\title{
Ensemble Machine Learning Forecaster for Day Ahead PV System Generation
}

\author{
Mahtab Kaffash \\ Department of Electrical Engineering, KU Leuven \\ EnergyVille, Genk \\ Leuven, Belgium \\ e-mail:mahtab.kaffash@kuleuven.be
}

\author{
Geert Deconinck \\ Department of Electrical Engineering, KU Leuven \\ EnergyVille, Genk \\ Leuven, Belgium \\ e-mail: geert.deconinck@kuleuven.be
}

\begin{abstract}
In this paper, the application of machine learning methods to predict the day ahead photovoltaic power generation in hourly intervals from the previous days, without using any exogenous data, have been studied. In order to select the relevant features, a random forest feature selection is used. This paper proposes a forecasting approach based on ensembles of artificial neural networks and support vector regression. The focus of this paper is on a single installed photovoltaic system, and in order to evaluate the performance of the proposed approaches, the measured data related to the photovoltaic installation on the roof of EnergyVille-1 is used. The results show that proposed approach can improve the accuracy of forecasting.
\end{abstract}

Keywords-artificial neural network; day ahead forecasting; ensemble forecasting; feature selection; support vector regression

\section{INTRODUCTION}

In recent decades, the penetration of photovoltaic systems (PV) has increased in Europe due to the European Union (EU) 20-20-20 targets and decrease in the installation cost of PV systems [1]. However, apart from the environmental benefits like decreasing air pollution, the large share of PV systems brings some serious challenges in scheduling and operation of power systems [2]. The main reason is that the power produced by a PV system is influenced by solar irradiation and weather conditions which makes PV power output highly variable. In order to improve the scheduling and operation performance of power systems, there is a need for an accurate estimation of output of PV system [3]. Depending on the application, the forecasting horizon can be different; from 5-min ahead to long-term forecasting.

Thanks to recent development in machine-learning (ML) methods, the statistical models have become popular in order to forecast time series. Also, in PV power forecasting, they become popular due to easy-to-use implementation and acceptable performance. The most popular methods, according to [4], to predict PV power are Artificial Neural Networks (ANNs) [5], [6], [7] and Support Vector Regression (SVR) [8], [9], [10]. In spite of the good performance of proposed methods, the majority of them rely on weather predictions (solar irradiation prediction, air temperature prediction, cloud coverage, etc.). However, those weather predictions are not

This project receives support from C24/16/018 project, founded by Internal Funds KU Leuven.

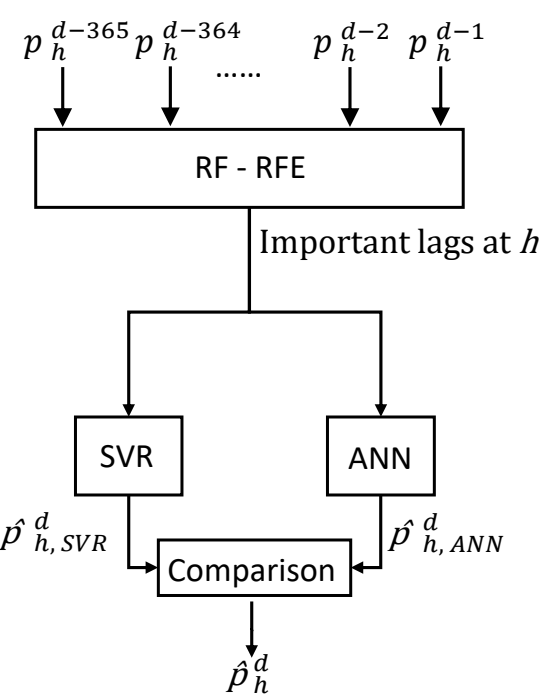

Figure 1. Overview of proposed approach.

always available and in some cases the data is not freely available [11].

The main idea of this paper is to develop a model for forecasting hourly day ahead PV production without using any exogenous inputs. In this situation, feature selection plays an important role to select the relevant historical data. In order to select the relevant features, random forest recursive feature elimination (RF-RFE) is used. In this paper two different machine-learning methods are used, SVR and ANN in order to predict the PV power. In order to increase the accuracy of the prediction an ensemble model which is a mixture of SVR and ANN is introduced. A general overview of the proposed approach is shown in Fig. 1.

The rest of the paper is organized as follows. First, the case study of this work is described in Section II. Then, in Section III the proposed methodology to forecast day ahead PV generation is presented. Section IV presents and discusses the simulation results and finally, in Section $\mathrm{V}$ the paper is concluded. 


\section{DATA}

\section{A. Case Study}

A 368-kWp PV system has been installed on the flat roof of the EnergyVille-1 building, a research center located in city of Genk in Belgium, in April 2016. This PV system includes 24 three-phase inverters and more than 20 sensors to measure the real time data related to the PV generation and weather conditions (global horizontal radiance, ambient air temperature, and wind speed). All the sensors and the inverters communicate with the logger and then the logger sends operational and weather data to ABB's online portfolio manager (AuroraVision) [12]. All the data are available for download as 5-min, 15-min, hourly, etc. averages.

In this paper, the data for total PV generation from $1^{\text {st }}$ May 2016 to $30^{\text {th }}$ January 2019 with hourly resolution have been downloaded from AuroraVision. Only the data from 6:00 am to $10: 00 \mathrm{pm}$ is considered, since most of the PV production values are zero out of this time interval. As a result, for each day there are 16 data points and there exist 1005 days between $1^{\text {st }}$ May 2016 and $30^{\text {th }}$ January 2019, which lead to 16080 data points available to forecast $\mathrm{PV}$ production.

Fig. 2 shows the heat map for the hourly PV production during 16 hours under considered time period. The significant changes in PV production are observable in Fig. 2, the highest hourly data is approximately $218 \mathrm{kWh}$ while the lowest is zero. This variation in data brings challenges for the forecasting task.

It should be mentioned that the PV panels have been installed in different four directions: South, North, East- and West-facing modules. After analyzing the measured data, it has been determined that the maximum PV generation reaches $60 \%$ of the installed capacity in an hourly average.

\section{B. Training Set, Validation Set and Test Set}

The available data set is divided into three sets: training, validation, and test. The splitting range is $60 \%, 20 \%$, and $20 \%$, respectively. The training set is used to train the models. Then, in ANN and SVR there are some variable parameters which require to be tuned. Therefore, validation set is needed to choose the optimal parameters and select the best model according to the evaluation metric. Afterwards, the test set is used to evaluate the accuracy of the selected model.

\section{PRoposed Approaches}

\section{A. Feature Selection}

As mentioned before, this paper attempts to forecast day ahead PV production with the resolution of 1 hour while using the historical data only, without using any exogenous data. As a result, the only available features are the past data for PV production. However, all the available features are not relevant, so the first step is to select the most relevant features, or lags in this case. Feature selection plays an important role in order to improve the accuracy of prediction model [13]. There are some researchers who applied the same lags for the whole time period of prediction. For instance, Yona et al. [3] considered the data related to the past 16 days from the day before a forecast day, and past 16 days before and after the forecast day in the previous year as the relevant features. However, this method would not work on Belgian data set since there is more uncertainty in weather.

In this paper, in order to rank the features a RF-RFE is applied. RF-RFE creates a predictive model and then ranks the features and finally prunes those with lower scores. In order to implement RF-RFE, to detect the relevant lags for hour $h$, in day $d$, all the data related to the PV production at $h$, from the last year, $p_{h}^{d-365}$, till the day before, $p_{h}^{d-1}$, are the input of RF-RFE, and the PV production of day $d$ at $h, p_{h}^{d}$, is the output of the RF-RFE (Fig. 1). Fig. 3 shows the selected lags for each hour.

As Fig. 3 illustrates the number of relevant features for each hour is different, the minimum required features belongs to 6:00 p.m. ( $\left(13^{\text {th }}\right.$ hour) with selected 8 relevant features while there are selected 22 relevant features for 4:00 p.m. ( $11^{\text {th }}$ hour). It should be mentioned that this procedure of feature selection is based on an average process, which means that although there is not a strong correlation between data from winter time and the data from summer time, in the concept of averaging the data from 6 months ago (the values around -180 in Fig. 3) has been detected as an relevant features.

\section{B. Feed-Forward Neural Network}

ANNs have become popular in different forecasting applications. There are many ways to construct an ANN, however in this paper a feed-forward neural network (FFNN) is used to predict the PV production for day ahead.

Fig. 4 shows the general architecture of the ANN algorithm built in this paper. The idea is to train separate ANN for each hour and as mentioned before, the number of relevant features can be different for each hour. As a result, there will be different input nodes depending on the number of features while there will be only one neuron in the output layer. A linear activation function (AF) is chosen for output layer, based on the concept of regression.

In this paper in order to have the best architecture for ANN, the number of neurons in hidden layer and the activation function in this layer are tuned during the learning process.

\section{Support Vector Regression}

SVR was introduced by Drucker et al. [14] to solve regression problems, which is a type of support vector machine (SVM). The basic idea of SVR is to non-linearly map the input vector $(p)$ into high-dimensional feature space and then perform linear regression in the feature space. So, the problem can be written as:

$$
\begin{gathered}
\min \quad \frac{1}{2}\|w\|^{2}+C \sum_{d=1}^{D} \epsilon_{d} \\
\text { s.t. } \quad \hat{p}_{d}\left(w \cdot \phi\left(p_{d}\right)+b\right) \geq 1-\epsilon_{d}, \\
\epsilon_{d} \geq 0, d=0, \ldots, D, \quad C>0
\end{gathered}
$$




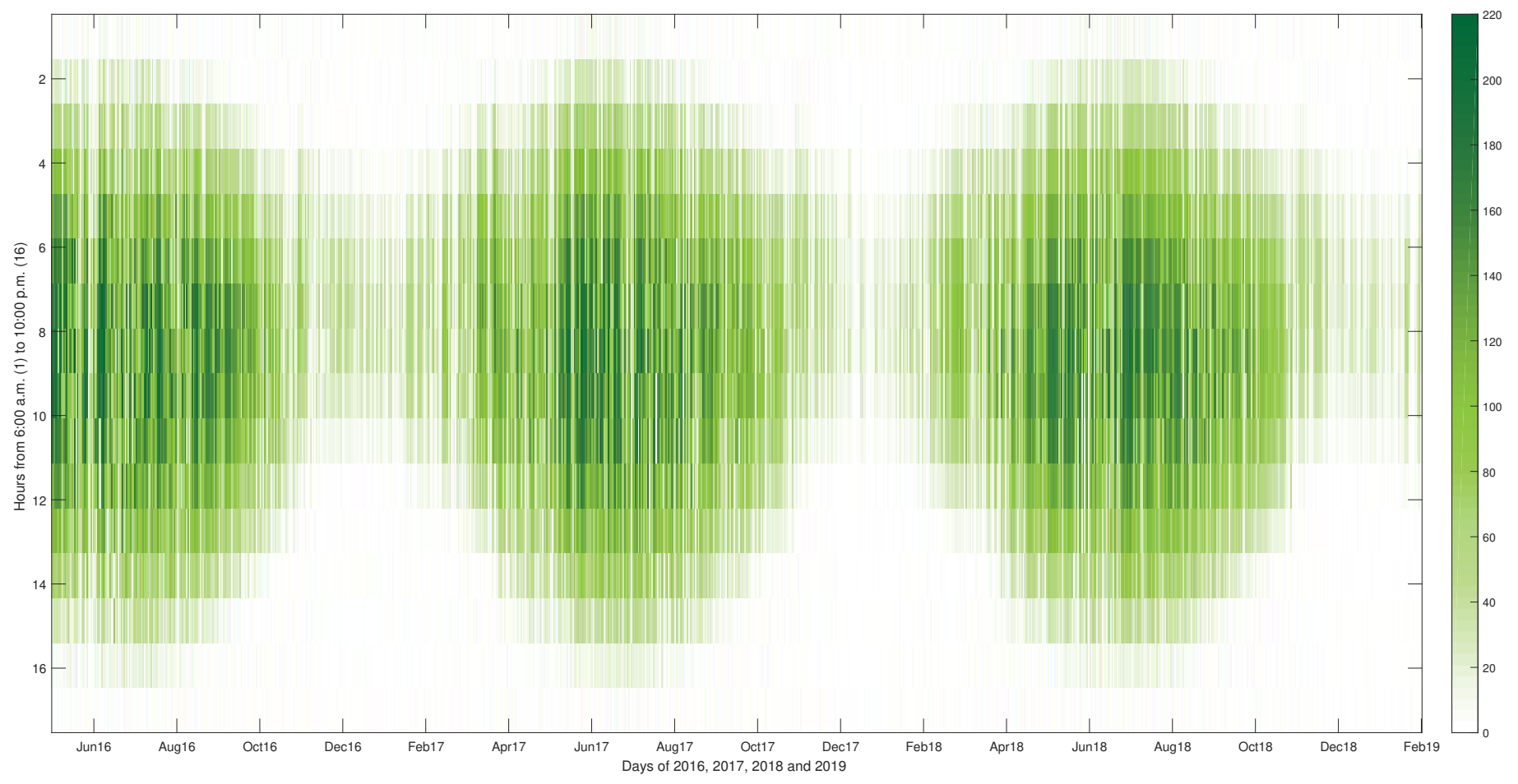

Figure 2. Overview of the PV generation (in kWh per hour) installed on the roof of EnergyVille-1, the amount of PV generation corresponds to the heat-map color bar.

where $C$ and $\epsilon$ are the hyperparameters of SVR, and $\phi($.$) is$ kernel function. As recommended in [15], since the distribution of our data is close to the normal, the following radial basis function (RBF) kernel (2) is used in this paper.

$$
\phi\left(p_{d}, p_{j}\right)=\exp \left(-\gamma\left\|p_{d}, p_{j}\right\|^{2}\right)
$$

where $\gamma$ is the RBF hyperparameter. As a result, in a SVR using $\mathrm{RBF}$ as kernel function there are three different hyperparameters that should be tuned in order to select the best predictor.

\section{Evaluation Metrics}

To analyze the accuracy of forecasting, a standard performance measure, Mean Relative Error (MRE) is used, as defined below:

$$
M R E=\frac{1}{D} \sum_{d=1}^{D}\left|\frac{p_{h}^{d}-\hat{p}_{h}^{d}}{R}\right| \times 100
$$

where $D$ in the number of instances (number of days in each data set) and $R$ is data range.

\section{Simulation Results}

The proposed method which is explained in Section III, is applied on the data related to PV installation on the roof of EnergyVille-1. The hourly data related to PV generation is available from $1^{\text {st }}$ May 2016 to $30^{\text {th }}$ January 2019. According to the feature selection process, we need data with one year lag $(d-365)$. As a result the data from $1^{\text {st }}$ May 2016 to $30^{\text {th }}$ April 2017 is only used as the inputs for feature selection, while the rest of data is used for ML training and test procedure as well. Therefore, the data set that becomes available as the target of ML is from $1^{\text {st }}$ May 2017 to $30^{\text {th }}$ January 2019. This means that for each hour there will be 640 instances available to build the forecaster.

As mentioned before, the data is divided in three categories: training, validation and test set with portion of $60 \%, 20 \%$ and $20 \%$, respectively. As a result, there are 384,128 , and 128 instances in training set, in validation set and in test set, respectively. One important thing to note here is the data shuffling before dividing the data. Since this data is measured through the time, there is a huge difference between the value for PV production during the summer and winter. If the model is built by taking samples from certain season, and then test it against another season, then the model would not perform well. So, we should randomize the order of data to make sure that the resulting three sub-groups have the same behavior. In other words, the data is shuffled between different days.

The next step is to select the optimal parameters for SVR and ANN by using grid search analysis. For ANN presented in Fig. 4, the hyperparameters are the number of neurons and the activation function in hidden layer. Number of neurons are set to $\{1,2, \ldots, 100\}$ and Sigmoid, Tanh, and Softplus function are 


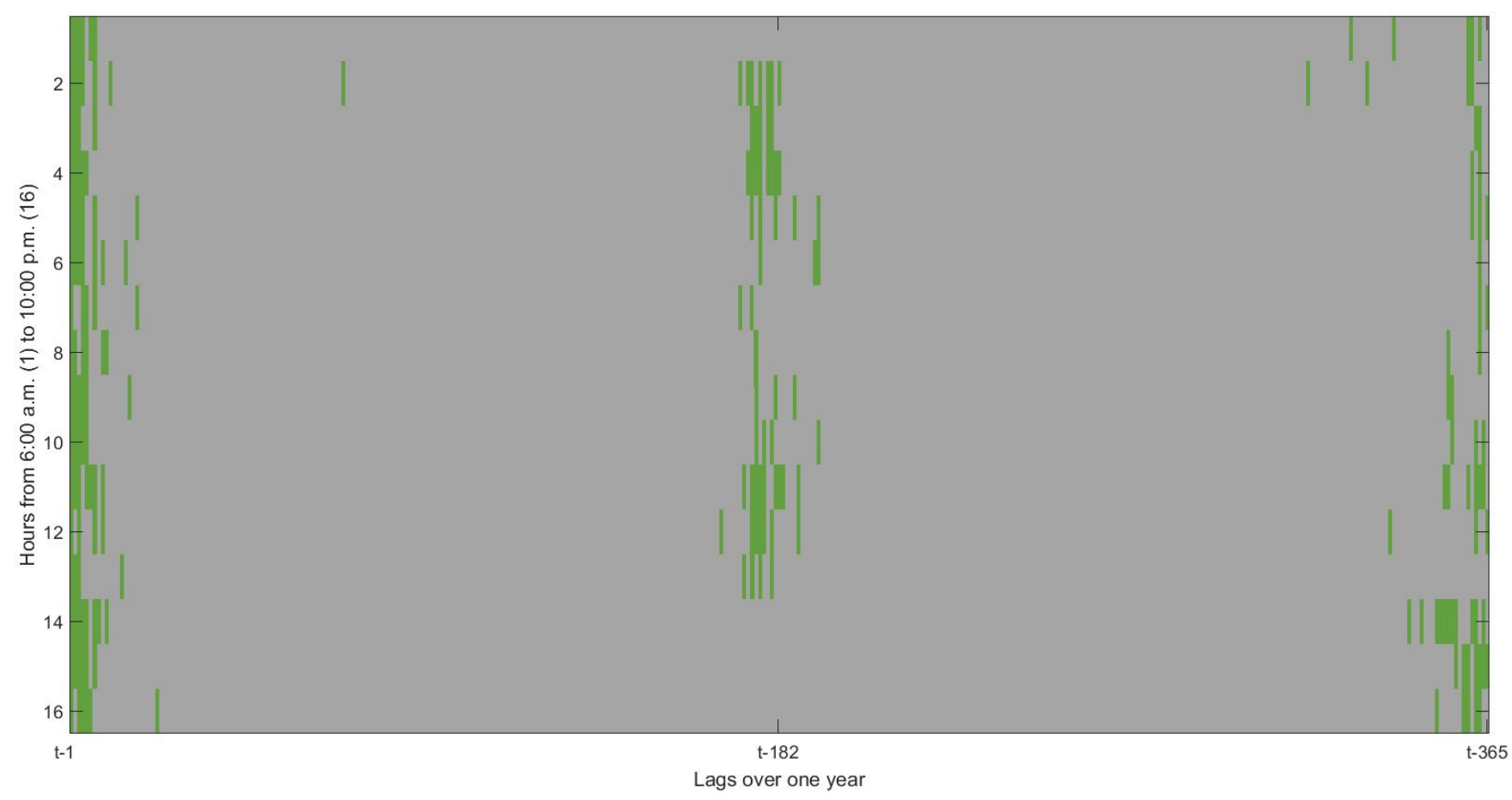

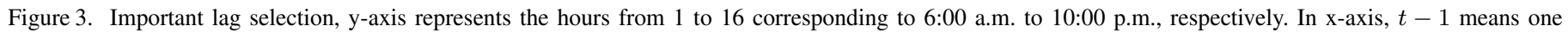
day ago and $t-365$ means one year ago. Green color represents selected lags.

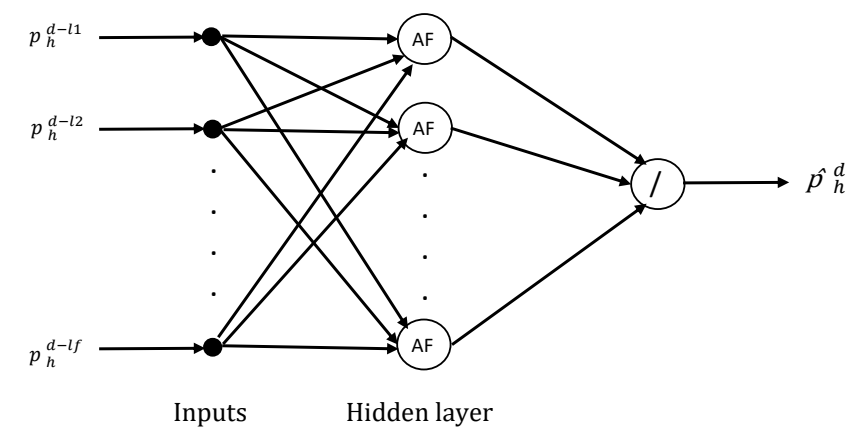

Figure 4. Architecture of a ANN for a single hour, the number of input features and number of neurons and activation function $(\mathrm{AF})$ for each hour are different.

analyzed by grid search. For SVR with RBF kernel, $C=\left\{10^{-2}\right.$, $\left.10^{-1}, \ldots, 10^{9}\right\}, \epsilon=\left\{10^{-9}, 10^{-8}, \ldots, 10^{0}\right\}, \gamma=\left\{10^{-9}, 10^{-8}\right.$, ..., $\left.10^{0}\right\}$ are selected.

In this phase, the hyperparameter candidates for ANN and SVR will be analyzed by grid search. According to the defined evaluation metrics, grid search will suggest the optimal hyperparameters for each hour and each method, separately. The best one, i.e. the one with the lowest error, is taken as the selected hyperparameter for the next step which is the comparison. For instance, $C=10^{8}, \epsilon=10^{-7}, \gamma=10^{-4}$ are the optimal SVR hyperparameters which are selected by grid search for PV forecaster at 11:00 a.m. and Sigmoid activation function with 32 neurons in hidden layer are selected for the optimal hyperparameters of ANN for 11:00 a.m. By comparing the MRE of ANN and the MRE of SVR, since the MRE of SVR is lower then SVR is selected as the final model to predict the data related to 11:00 a.m.

The basic idea is to train the model based on training data set and then evaluate the proposed model with validation set. As a result, in order to build the ensemble forecasting including SVR and ANN, the MRE of these two methods are compared to each other, using validation data set. Fig. 5 shows the MRE of the SVR and ANN running on validation set. As it can be seen from Fig. 5, from considered 16 hours, for 13 of them, SVR has a better performance, while only for three hours - the $3^{\text {rd }}, 9^{\text {th }}$, and $11^{\text {th }}$ hour; 8:00 a.m., 2:00 p.m., and 4:00 p.m., respectively- ANN performs better. As a result, in order to increase the accuracy of the forecasting, for those three hours, ANN is chosen and for the other hours SVR is used.

In order to evaluate, the proposed method is applied on test set data. Fig. 6 illustrates the MRE of proposed method compared with the models when there is only ANN or SVR. As it can be seen, the proposed method has better performance. The possible reason that ANN, has better performance over those three hours can be explained by variation in the data in those hours and also the number of features.

However, the computational cost of ensemble model is higher than a single ANN or single SVR, but it can improve the MRE up to $0.5 \%$ and $0.08 \%$ compared to single ANN and single SVR, respectively, in average for the whole time 


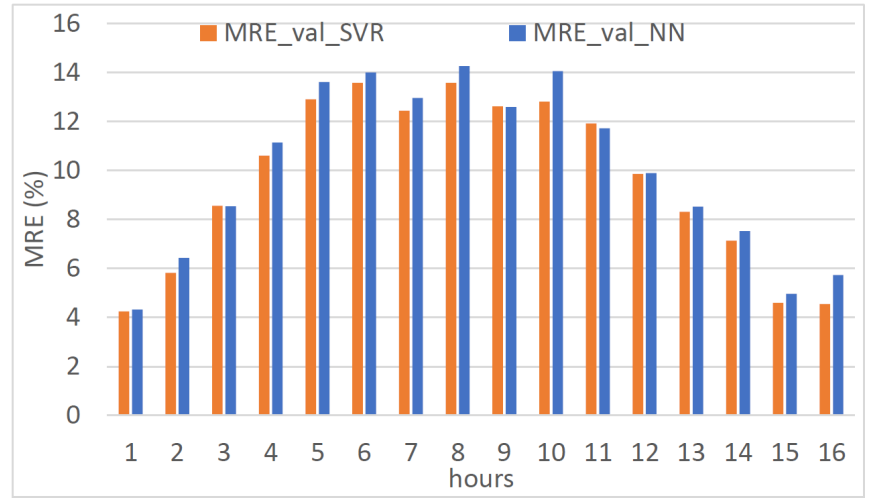

Figure 5. Accuracy of ANN and SVR on validation set.

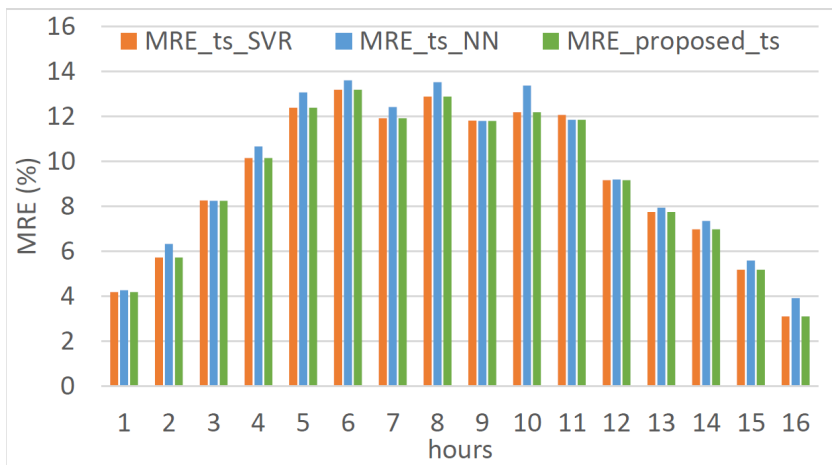

Figure 6. Accuracy of proposed method on test set.

period of test set (according to Fig. 6). It should be noted that although in scale of percentage these differences are small values, in terms of $\mathrm{kWh}$ it makes a differences. Also, compared to the results from other researchers, reaching to MRE, 9.15\% in average via proposed method counts as an advantage.

In order to compare the results of this paper with other researchers, Mellit et al. [16], Shi et al. [9], and Yang et al. [17] by considering the weather forecasting values, were able to reach to the lower MRE (less than 4\%) in average in order to forecast PV production. On the other hand, by comparing the results of this paper with [5], in which a PV forecaster independent from the weather forecasting values was built, a $17.24 \%$ MRE in average was obtained. Simulation results show that the proposed method can improve the performance of the PV power generation forecasting.

\section{CONCLUSIONS}

This paper proposes the power output forecasting for PV system based on ensemble machine learning methods, SVR and ANN by using RF-RFE to select the relevant features. The merit of the proposed method is that it does not require any exogenous data while in majority of literature, they used some external data which is not available for all the case studies. Simulation results show that in most of the time periods, SVR has better results than ANN. However, there are three hours that ANN performs better. As a result, in order to increase the accuracy, an ensemble model is proposed.
Although the computational cost of the ensemble method is higher than single SVR and single ANN, it can improve the accuracy of forecasting compared to the mentioned past researches.

\section{REFERENCES}

[1] B. V. Mbuwir, M. Kaffash, and G. Deconinck, "Battery scheduling in a residential multi-carrier energy system using reinforcement learning," 2018 IEEE International Conference on Communications, Control, and Computing Technologies for Smart Grids (SmartGridComm), pp. 1-6, Denmark, October 2018.

[2] E. G. Kardakos, M. C. Alexiadis, S. I. Vagropoulos, C. K. Simoglou, P. N. Biskas, and A. G. Bakirtzis, "Application of time series and artificial neural network models in short-term forecasting of PV power generation," 48th IEEE International Universities' Power Engineering Conference (UPEC), pp. 1-6, September 2013.

[3] A. Yona, T. Senjyu, A. Y. Saber, T. Funabashi, H. Sekine, and C. H. Kim, "Application of neural network to 24-hour-ahead generating power forecasting for PV system,” 2008 IEEE Power and Energy Society General Meeting-Conversion and Delivery of Electrical Energy in the 21st Century, pp. 1-6, July 2008.

[4] S. Sobri, S. K. Kamali, and N. A. Rahim, "Solar photovoltaic generation forecasting methods: A review," Energy Conversion and Management, 156, pp. 459-497, January 2018.

[5] M. Rana, I. Koprinska, and V. G. Agelidis, "Forecasting Solar Power Generated by Grid Connected PV Systems Using Ensembles of Neural Networks," 2015 International Joint Conference on Neural Networks (IJCNN), pp. 1-8, July 2015.

[6] A. Dolara, S. Leva, M. Mussetta, and E. Ogliari, "PV hourly dayahead power forecasting in a micro grid context," 16th International Conference on Environment and Electrical Engineering (EEEIC), pp. 1-5, June 2016.

[7] E. E. Roussineau, P. Otto, and P. Gratzfeld, "Short-Term Forecasting in Electric Power Systems using Artificial Neural Networks," Innovative Smart Grid Technologies Conference Europe (ISGT-Europe), pp. 1-6, October 2018.

[8] B. Wolff, J. Kühnert, E. Lorenz, O. Kramer, and D. Heinemann, "Comparing support vector regression for PV power forecasting to a physical modeling approach using measurement, numerical weather prediction, and cloud motion data," Solar Energy, 135, pp. 197-208, 2016.

[9] J. Shi, W. J. Lee, Y. Liu, Y. Yang, and P. Wang, "Short-Term Forecasting in Electric Power Systems using Artificial Neural Networks," IEEE Transactions on Industry Applications, vol. 48, no. 3, pp. 1064-1069, May 2012.

[10] M. Hossain, S. Mekhilef, M. Danesh, L. Olatomiwa, and S. Shamshirband, "Application of extreme learning machine for short term output power forecasting of three grid-connected PV systems Monowar," Journal of Cleaner Production, 167, pp. 395-405, 2017.

[11] H. T. C. Pedro, and C. F. M. Coimbra, "Assessment of forecasting techniques for solar power production with no exogenous inputs," Solar Energy 86.7, pp. 2017-2028, January 2012.

[12] G. Yordanov, F. Smolders, A. Olaerts, G. Verbeek, K. Baert, and J. Driesen, "A 368-kWp Grid-connected PV System: Known and Hidden Losses," European PV Solar Energy Conference and Exhibition (EUPVSEC), pp. 1-6, Germany, September 2017.

[13] M. A. Hall, and L. A. Smith, "Feature selection for machine learning: Comparing a correlation-based filter approach to the wrapper," The Florida Artificial Intelligence Research Society (FLAIRS) Conference, pp. 235-239, May 1999.

[14] H. Drucker, C. J. C. Burges, L. Kaufman, A. Smola, and V. Vapnik, "Support vector regression machines," Advances in neural information processing systems, pp. 155-161, 1997.

[15] S. Ali, and K. A. Smith, "Automatic Parameter Selection for Polynomial Kernel," Proceedings Fifth IEEE Workshop on Mobile Computing Systems and Applications, pp. 243-249, October 2003.

[16] A. Mellit, S. Sağlam, and S. A. Kalogirou, "Artificial neural networkbased model for estimating the produced power of a photovoltaic module," Renewable Energy 60, pp. 71-78, December 2013.

[17] H. T. Yang, C. M. Huang, Y. C. Huang, and Y. S. Pai, "A WeatherBased Hybrid Method for 1-Day Ahead Hourly Forecasting of PV Power Output," IEEE Transactions on Sustainable Energy, vol. 5, no. 3, pp. 917-926, July 2014. 